Milan Matejdes, Department of Mathematics, Technical University in

Zvolen, Masarykova 24, 96053 Zvolen, Slovakia,

e-mail:matejdes@vsld.tuzvo.sk

\title{
CONTINUITY POINTS OF FUNCTIONS ON PRODUCT SPACES
}

\begin{abstract}
The paper is devoted to joint and separate connectivity properties of functions on product spaces. Examples, generalized types of continuity and quasicontinuity points of separately connected functions are studied.
\end{abstract}

\section{Introduction and Preliminary Examples}

Many authors consider the notion of local $w^{*}$ continuity as a suitable tool for investigation of relationship between continuity and connectivity [3], [6], [7], [10]. There arises a question whether it is possible to define a point version of local $w^{*}$ continuity. Another motivation of this paper is to find the weakest assumptions on spaces $X, Y$ and $Z$ as well as on continuity types of the sections $f_{x}$ and $f_{y}$ of a function $f: X \times Y \rightarrow Z$ such that $f$ has at least one point of joint continuity [9]. From this point of view it seems to be closely related to the following properties of functions:

- quasi and almost continuity,

- O-connectedness,

- cliquishness,

- local $w^{*}$ continuity.

Key Words: quasicontinuity, connectivity, separation properties

Mathematical Reviews subject classification: 26A15

Received by the editors February 1, 1997

* Supported by VGA Research Grant GD-37 
The notion of O-connectedness was introduced in [6] dealing with a decomposition of continuity and separate properties of functions. Since this notion as well as the quasi continuity, almost continuity and cliquishness are closely related to the problems mentioned above we recall them. In the sequel $X, Y, Z$ are topological spaces and $(M, d)$ is a metric space.

Definition 1. - A function $f: X \rightarrow Y$ is quasi continuous (almost continuous) at a point $x$ if for any open set $V$ containing $f(x), x \in$ $\mathrm{cl}\left(\operatorname{int}\left(f^{-1}(V)\right)\right)\left(x \in \operatorname{int}\left(\operatorname{cl}\left(f^{-1}(V)\right)\right)\right)$, where int $(A)$ and $\operatorname{cl}(A)$ denote the interior and closure of a set $A[4],[2]$.

- A function $f: X \rightarrow M$ is cliquish at a point $x$ if for any $\varepsilon>0$ and any open set $U$ containing $x$ there is a non-empty open set $H \subset U$ such that $d\left(f\left(x_{1}\right), f\left(x_{2}\right)\right)<\varepsilon$ for any $x_{1}, x_{2} \in H[11]$.

- A function $f$ is quasi continuous, almost continuous, cliquish if it is so at any point.

- A function $f: X \rightarrow Y$ is O-connected if $f(V)$ is connected for any open connected set $V \subset X[6]$.

- A function $f: X \rightarrow Y$ is locally $w^{*}$ continuous if there is a base $\vartheta$ for $Y$ such that $f^{-1}(\operatorname{Fr}(V))$ is closed for any $V \in \vartheta$, where $\operatorname{Fr}(V)$ is boundary of $V[7]$.

The goal of this paper is to introduce a notion of continuity being a quasi and point version of local $w^{*}$ continuity.

Definition 2. A function $f: X \rightarrow Y$ is quasi $w^{*}$ continuous at a point $x$ if for any open set $V$ containing $f(x)$ there is an open set $H \subset V$ containing $f(x)$ and there is a quasi open set $U$ containing $x$ such that $U \cap f^{-1}(\operatorname{Fr}(H))=\emptyset$, where a set $A$ is called quasi open if $A \subset \operatorname{cl}(\operatorname{int}(A))$. A function $f$ is quasi $w^{*}$ continuous if it is so at any point.

It is clear, if $f$ is locally $w^{*}$ continuous, then $f$ is quasi $w^{*}$ continuous.

Example 1. A function $f:[0, \infty) \rightarrow \mathbb{R}$ defined as $f(0)=0$ and $f(x)=\sin \frac{1}{x}$ for $x>0$ is quasi $w^{*}$ continuous but it is not locally $w^{*}$ continuous at zero.

For further investigation the following construction of a function being quasi $w^{*}$ continuous at no point will be useful.

Example 2. Let $\left\{A_{\alpha}\right\}_{\alpha \in I}$ be decomposition of $\mathbb{R}$ into $c$ pairwise disjoint sets $A_{\alpha}$ each of which is dense in $\mathbb{R}$, where $I$ is index set of cardinality c. Define $f: \mathbb{R} \rightarrow \mathbb{R}$ by $f(x)=f_{0}(\alpha), x \in A_{\alpha}$ where $f_{0}: I \rightarrow \mathbb{R}$ is one-to-one function 
mapping $I$ onto $\mathbb{R}$. Function $f$ is not quasi $w^{*}$ continuous at any point and it is almost continuous and O-connected.

Considering a function $f: X \times Y \rightarrow Z$ we denote by $f_{x}: Y \rightarrow Z$ and $f_{y}: X \rightarrow Z x$-section and $y$-section of $f$ given by $f_{x}(y)=f(x, y)$ and $f_{y}(x)=f(x, y)$, respectively. If all $x$-sections and all $y$-sections of $f$ are quasi $w^{*}$ continuous (O-connected) we say $f$ is separately quasi $w^{*}$ continuous (separately O-connected).

As the following two examples show there is no connection between separate quasi $w^{*}$ continuity and quasi $w^{*}$ continuity.

Example 3. There is a real function $f$ defined on $X=[a, b] \times[c, d]$ being separately quasi $w^{*}$ continuous which is not quasi $w^{*}$ continuous.

Proof. Let $A$ be a set of second category such that no three points from $A$ are collinear [8, Th. 15.5]. Since int $(D(A))$ is non-empty $(D(A)$ is a set of all points at which $A$ is of second category), there are intervals $[a, b],[c, d]$ such that $[a, b] \times[c, d] \subset \operatorname{int}(D(A))$. Put $X=[a, b] \times[c, d]$. Let $\left\{V_{n}\right\}_{n=1}^{\infty}$ be a base of $X$. Let $A_{0}=\left\{a_{0}^{n}\right\}_{n=1}^{\infty}$, where $a_{0}^{n} \in V_{n} \cap A$. By transfinite induction, define $A_{\alpha}=\left\{a_{\alpha}^{n}\right\}_{n=1}^{\infty}$, where $a_{\alpha}^{n} \in V_{n} \cap\left(A \backslash \bigcup_{\beta<\alpha} A_{\beta}\right)$. Note, $V_{n} \cap A$ is of second category and $\bigcup_{\beta<\alpha} A_{\beta}$ is of first category for any countable ordinal number $\alpha$. It is clear $A_{\alpha}$ is dense in $X$. Hence $\left\{A_{\alpha}\right\}_{\alpha \in I}$ is a system of disjoint subsets of $A$ each of which is dense in $X$, where $I$ is index set of cardinality $c$.

Define $f: X \rightarrow \mathbb{R}$ by

$$
f(x)= \begin{cases}f_{0}(\alpha), & x \in A_{\alpha} \\ 0, & x \in X \backslash \bigcup_{\alpha \in I} A\end{cases}
$$

where $f_{0}$ is one-to-one function mapping $I$ onto $\mathbb{R}$.

Function $f$ is not quasi $w^{*}$ continuous, since $f^{-1}(S)$ is dense in $X$ for any non-empty set $S \subset \mathbb{R}$. Each section of $f$ takes non-zero value at most two points, hence $f$ is separately quasi $w^{*}$ continuous.

Example 4. There is a function $g: \mathbb{R} \times \mathbb{R} \rightarrow \mathbb{R}$ which is quasi $w^{*}$ continuous but not separately quasi $w^{*}$ continuous.

Define $g: \mathbb{R} \times \mathbb{R} \rightarrow \mathbb{R}$ as follows

$$
g(x, y)= \begin{cases}f(y), & x=0 \text { where } f \text { is from Example } 2 \\ 0, & x \neq 0\end{cases}
$$




\section{Main Results}

Lemma 1. If $f: X \times Y \rightarrow Z$ is separately O-connected, then $f(U \times V)$ is connected for any open connected sets $U \subset X$ and $V \subset Y$.

Proof. Let $z_{1}=f\left(\left[x_{1}, y_{1}\right]\right), z_{2}=f\left(\left[x_{2}, y_{2}\right]\right) \in f(U \times V)$. Since $f_{x_{1}}$ and $f_{y_{2}}$ are O-connected, then $f_{x_{1}}(V)=f\left(\left\{x_{1}\right\} \times V\right)$ and $f_{y_{2}}(U)=f\left(U \times\left\{y_{2}\right\}\right)$ are non-empty connected sets containing $f\left(\left[x_{1}, y_{2}\right]\right)$. That means arbitrary two points $z_{1}, z_{2} \in f(U \times V)$ can be joined by a connected set, hence $f(U \times V)$ is connected.

Theorem 1. Let $X, Y$ be locally connected spaces. If $f: X \times Y \rightarrow Z$ is separately O-connected, then $f$ is O-connected.

Proof. It is modification that of [6, Th. 1]. Let $G$ be a connected open subset of $X \times Y$. Then $G$ can be expressed as a union of a collection of basis elements of the form $U \times V$, where $U$ and $V$ are open and connected. Let $z_{1}=f\left(\left[x_{1}, y_{1}\right]\right), z_{2}=f\left(\left[x_{2}, y_{2}\right]\right) \in f(G)$. The points $\left[x_{1}, y_{1}\right],\left[x_{2}, y_{2}\right]$ can be joined by a finite collection $U_{1} \times V_{1}, U_{2} \times V_{2}, \ldots, U_{n} \times V_{n}$ of such basis elements, such that $\left[x_{1}, y_{1}\right] \in U_{1} \times V_{1}$ and $\left[x_{2}, y_{2}\right] \in U_{n} \times V_{n}$ and any two successive sets $U_{i} \times V_{i}, U_{i+1} \times V_{i+1}$ have at least one common point. Since $U_{i} \times V_{i}$, $i=1,2, \ldots, n$ are open connected, $f\left(U_{i} \times V_{i}\right)$ are connected, by Lemma 1 . Since $z_{1} \in f\left(U_{1} \times V_{1}\right), z_{2} \in f\left(U_{n} \times V_{n}\right)$ and $f\left(U_{i} \times V_{i}\right) \cap f\left(U_{i+1} \times V_{i+1}\right) \neq \emptyset$ for any $i=1, \ldots, n-1, z_{1}$ and $z_{2}$ can be joined by a connected set. Since $z_{1}, z_{2}$ are arbitrary points from $f(G), f(G)$ is connected.

The next theorem deals with sufficient conditions for quasi continuity under slight conditions on $X$ and $Z$. The space $X$ is supposed to be of $\pi$-connected type; i.e., each open non-empty subset of $X$ contains an open non-empty connected subset.

Theorem 2. Let $X$ be of $\pi$-connected type and $f: X \rightarrow Z$ be $O$-connected. If $f$ is almost continuous at $x$ and quasi $w^{*}$ continuous at $x$, then $f$ is quasi continuous at $x$.

Proof. Let $U, V$ be open neighborhood of $x, f(x)$, respectively. Since $f$ is quasi $w^{*}$ continuous at $x$, there is an open set $H \subset V, f(x) \in H$ and there is a quasi open set $U_{0} \subset U$ containing $x$, such that $U_{0} \cap f^{-1}(\operatorname{Fr}(H))=\emptyset$. Since $f$ is almost continuous at $x$, there is an open set $U_{1} \subset U$ containing $x$ such that $f^{-1}(H)$ is dense in $U_{1}$. That means, the set $f^{-1}(H) \cap U_{0} \cap U_{1}$ is non-empty. The space $X$ is of $\pi$-connected type, hence there is a non-empty and connected set $U_{2} \subset U_{0} \cap U_{1} \subset U$ and $f\left(U_{2}\right)$ is connected. We claim $f\left(U_{2}\right) \subset H$. Assume there exists $x_{1} \in U_{2}$ such that $f\left(x_{1}\right) \notin H$. Since $U_{0} \cap f^{-1}(\operatorname{Fr}(H))=\emptyset, f\left(x_{1}\right) \notin$ 
$\operatorname{cl}(H)$. The set $f^{-1}(H)$ is dense in $U_{1}$, hence there exists $x_{2} \in U_{2}$ such that $f\left(x_{2}\right) \in H$. That means, $f\left(U_{2}\right)$ is non-connected, what is a contradiction.

Corollary 1. Let $X$ be of $\pi$-connected type and $Z$ be a second countable space. If $f: X \rightarrow Y$ is $O$-connected, then $f$ is quasi $w^{*}$ continuous except for a set of first category if and only if $f$ is quasi continuous except for a set of first category.

Proof. The proof follows from [12, Th. 1], since $f$ is almost continuous except for a set of first category.

Corollary 2. Let $X$ be Baire of $\pi$-connected type and $Z$ be a separable metric space. If $f$ is $O$-connected, then $f$ is cliquish if and only if $f$ is quasi $w^{*}$ continuous except for a set of first category.

Proof. Implication " $\Rightarrow$ " follows from [1].

$" \Leftarrow "$ By Corollary $1, f$ is quasi continuous on a dense set $P$, hence $f$ is cliquish on $P$. Since the set of all points at which $f$ is cliquish is closed, $f$ is cliquish at any point.

As for the continuity assumptions in Theorem 2 none of them can be omitted.

Example 2 shows that quasi $w^{*}$ continuity is essential. Dirichlet function shows that O-connectedness cannot be omitted. Next example completes that almost continuity is essential too.

Example 5. Define $f: \mathbb{R} \rightarrow \mathbb{R}$ as

$$
f(x)= \begin{cases}1, & x \in \bigcup_{n=1}^{\infty}\left(\frac{1}{n+1}, \frac{1}{n}\right) \\ 0, & x=0 \\ f_{0}(\alpha), & n \neq 0 \text { and } x \notin \bigcup_{n=1}^{\infty}\left(\frac{1}{n+1}, \frac{1}{n}\right)\end{cases}
$$

where $f_{0}$ and $\left\{A_{\alpha}\right\}_{\alpha \in I}$ are from Example 1 .

Function $f$ is $\mathrm{O}$-connected, quasi $w^{*}$ continuous at $x=0$ and it is not almost continuous at $x=0$.

Theorem 3. Let $X, Y$ be locally connected spaces and $Z$ be second countable. If $f: X \times Y \rightarrow Z$ is separately O-connected, then $f$ is quasi $w^{*}$ continuous except for a set of first category, if and only if $f$ is quasi continuous except for a set of first category. 
Proof. Since $X \times Y$ is locally connected, the proof follows from Corollary 1 and Theorem 1.

A $\pi$-base for a space $(Y, \mathcal{T})$ is a subset $\mathcal{P}$ of $\mathcal{T}-\{\emptyset\}$ such that every nonempty set $U$ of $\mathcal{T}$ contains a non-empty set $G$ of $\mathcal{P}[9] .(Y, \mathcal{T})$ is locally of $\pi$-countable connected type if each open non-empty subset of $Y$ contains an open connected non-empty subset having a countable $\pi$-base.

Theorem 4. Let $X$ be a Baire space, $Y$ be Baire locally of $\pi$-countable connected type and $Z$ be separable matric. If $f_{x}$ is O-connected and quasi $w^{*}$ continuous except for a set of first category for any $x \notin S(S \subset X$ of first category) and $f_{y}$ is quasi continuous for any $y \in Y$, then $f: X \times Y \rightarrow Z$ is cliquish. Moreover, if $X \times Y$ is Baire, $f$ is continuous on a residual set.

Proof. It follows from Corollary 2 and [5, Th. 3, Cor. 1].

\section{References}

[1] L. A. Fudali, On cliquish functions on product spaces, Math. Slovaca, 33 (1983), 53-58.

[2] T. Husain, Almost continuous mappings, Prace Matem., 10 (1966), 1-7.

[3] M. Levine, A decomposition of continuity in topological spaces, Amer. Math. Monthly, 68 (1961), 44-46.

[4] S. Marcus, Sur les fonctions quasicontinues an sens de S. Kempisty, Colloq. Math 8 (1961), 47-53.

[5] M. Matejdes, Quasi-continuous and cliquish selections of multifunctions on product spaces, Real Anal. Exchange, 18 (1992-93), 214-220.

[6] R. Mimna, A note on separate continuity and connectivity properties, Math. Bohemica, 33 (1997), 57-61.

[7] R. Mimna and D. Rose, A note on closed graph functions and local $w^{*}$ continuity, Real Anal. Exchange, 18 (1992-93), 549-552.

[8] J. Oxtoby, Measure and category, New York - Heidelberg - Berlin, 1971.

[9] Z. Piotrowski and E. Wingler, A note on continuity points of functions, Real Anal. Exchange 16 (1990-91), 408-414.

[10] D. Rose, On Levine's decomposition of continuity, Canad. Math. Bull. 21(4) (1978), 477-480. 
[11] H. P. Thielman, Types of functions, Amer. Math. Monthly 60 (1953), 156-161.

[12] M. Wilhelm, Nearly lower semicontinuity and its applications, General Topology and its Relations to Modern Analysis and Algebra, V. Proc. Fifth Prague Topol. Symp. 1981, Berlin, 1983, pp. 692-698. 